\title{
Orbital Metastasis as the Initial Presentation of Invasive Lobular Carcinoma of Breast
}

\author{
Yutaka Tomizawa $^{1}$, Rebecca Ocque ${ }^{2}$ and N. Paul Ohori ${ }^{2}$
}

\begin{abstract}
This is an unusual case of invasive lobular carcinoma of the breast presenting as an orbital metastasis. A 70 year-old female presented with a gradually worsening blurred vision, periorbital swelling and ascites. The biopsy of the eyelid demonstrated dense fibrosis with neoplastic cells and the diagnosis of carcinoma was made; however, the site of origin of carcinoma was difficult to determine. The histopathologic characteristics of the carcinoma in the orbit and ascites fluid combined with the immunophenotypic features helped determine the primary site of the malignancy. Subsequently, the primary malignancy was identified by examination of the patient's breast.
\end{abstract}

Key words: invasive lobular carcinoma of the breast, orbital metastasis, E-cadherin, estrogen receptor

(Intern Med 51: 1635-1638, 2012)

(DOI: 10.2169/internalmedicine.51.7641)

\section{Introduction}

We encountered an unusual case of invasive lobular carcinoma of the breast presenting as an orbital metastasis. The histopathologic characteristics of the carcinoma in the orbit and ascites fluid combined with the immunophenotypic features helped determine the primary site of the malignancy. Subsequently, the primary malignancy was identified by examination of the patient's breast.

\section{Case Report}

A 70-year-old woman with a past medical history of basal cell carcinoma of the right eyelid resected twenty years previously presented with a 9-month history of gradually worsening blurred vision, periorbital swelling, and redness in the right eye. During the month prior to presentation, she also experienced fatigue and increasing abdominal girth. She denied fever, headache, nausea, emesis, or change in bowel habits. Her previous cancer screening tests consisting of mammography and colonoscopy were negative. Physical examination at the time of presentation revealed right periorbital erythema and edema, conjunctival and scleral injection, decreased visual acuity, and significantly restricted right extraocular movements in all directions. The pupils were equal and reactive to light, bilaterally and there was no temporal tenderness or exophthalmos. Further ophthalmologic examination showed normal intraocular pressure and normal retina. Ascites was noted but the remaining physical examination was otherwise unremarkable. Laboratory studies showed hypoalbuminemia of $2.6 \mathrm{gm} / \mathrm{dL}$, but no liver dysfunction or coagulopathy. Magnetic resonance imaging (MRI) of the brain showed a diffuse abnormal enhancement involving the right orbit and extra-ocular muscles. However, the right optic nerve was normal in size and the globe was otherwise unremarkable (Fig. 1). The differential diagnosis included severe inflammatory response, lymphoma, primary or metastatic neoplasia. The biopsy of the right eyelid was initially submitted to the Hematopathology Division where the possibility of lymphoma was excluded. Histologically, the biopsy demonstrated dense fibrosis with neoplastic cells showing occasional intracytoplasmic vacuoles infiltrating throughout the collagen bundles. (Fig. 2). Immunohistochemical studies showed positive immunoreactivity to pancytokeratin. The diagnosis of carcinoma was made; however, the site of origin of carcinoma was not yet determined. A diagnostic paracentesis performed shortly afterwards revealed metastatic carci-

${ }^{1}$ Department of Medicine, University of Pittsburgh Medical Center, USA and ${ }^{2}$ Department of Pathology, University of Pittsburgh Medical Center, USA

Received for publication March 1, 2012; Accepted for publication March 5, 2012

Correspondence to Dr. Yutaka Tomizawa, tomizaway@upmc.edu 


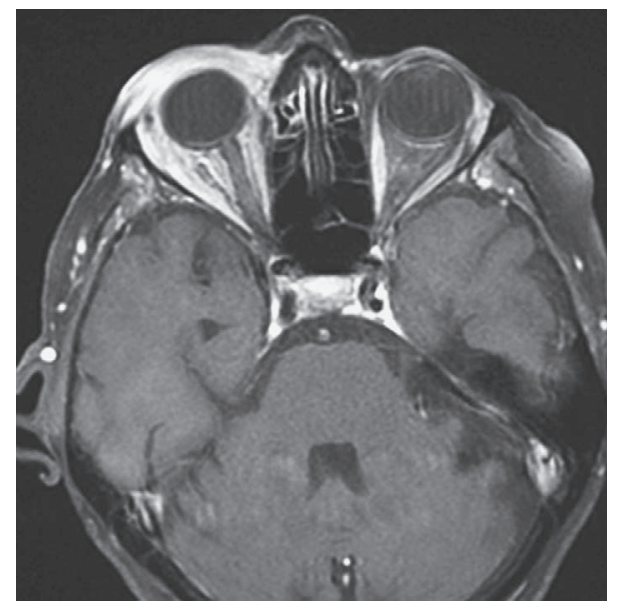

Figure 1. MRI of the Brain. Diffuse abnormal enhancement involving the right orbit and extra-ocular muscles. The right optic nerve is normal in size, and the globe is unremarkable. The left orbit and globe are unremarkable.

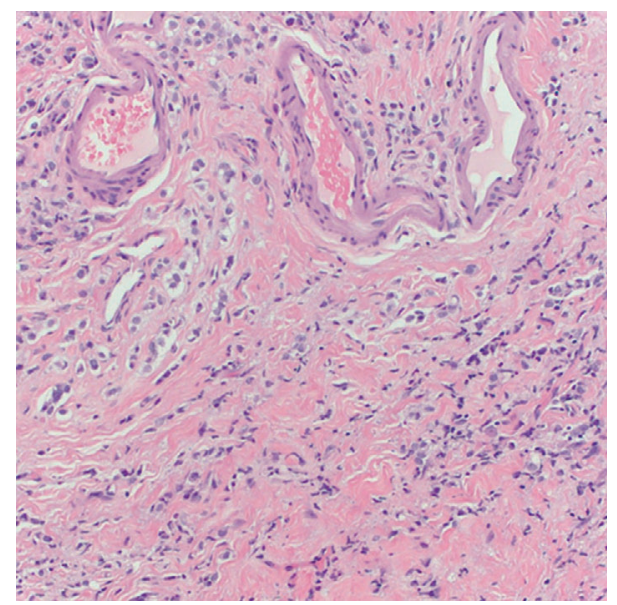

Figure 2. Hematoxylin and Eosin staining, Right Eyelid Cutaneous Biopsy. Infiltration of neoplastic cells with histiocytic and monocytic appearance with occasional intracytoplasmic mucosecretion and prominent scirrhous reaction.

noma cells with high nuclear to cytoplasmic ratio. Given the cytomorphologic appearance, the possibility of breast carcinoma was raised. Immunohistochemical studies demonstrated cytokeratin, estrogen receptor and mammoglobulin positivity and E-cadherin, progesterone receptor and HER-2/ NEU negativity in the neoplastic cells. The results were in keeping with the possibility of metastatic breast carcinoma, in particular lobular carcinoma. The orbital biopsy was reexamined and found to have similar immunophenotypic features (Fig. 3, 4). Upon repeat breast examination, the oncologist found a $1 \mathrm{~cm}$, hard, immobile mass in the right upper outer quadrant of the patient's breast. Mammogram demonstrated scattered fibroglandular tissue and delineated an area of architectural distortion within the upper outer quadrant of the right breast. Ultrasonography showed two hypoechoic lesions (largest lesion measuring $0.8 \mathrm{~cm}$ ) with irregular margins. Additional smaller lesions and two enlarged axillary lymph nodes were also identified. Ultrasound

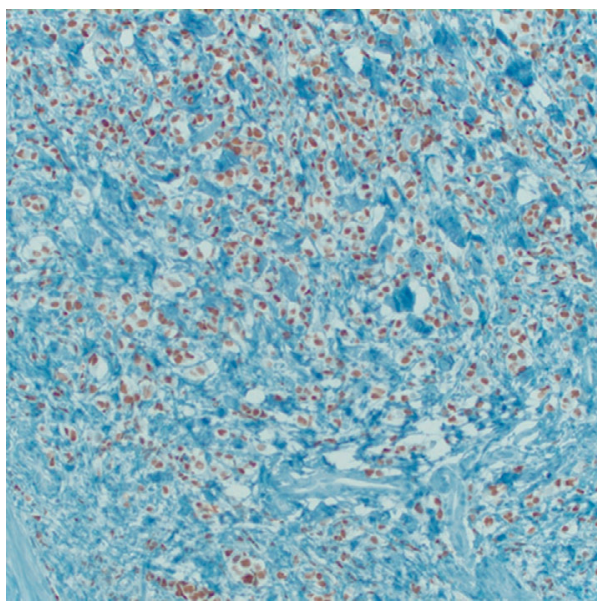

Figure 3. Immunohistochemical Stain, Positive for Estrogen Receptor.

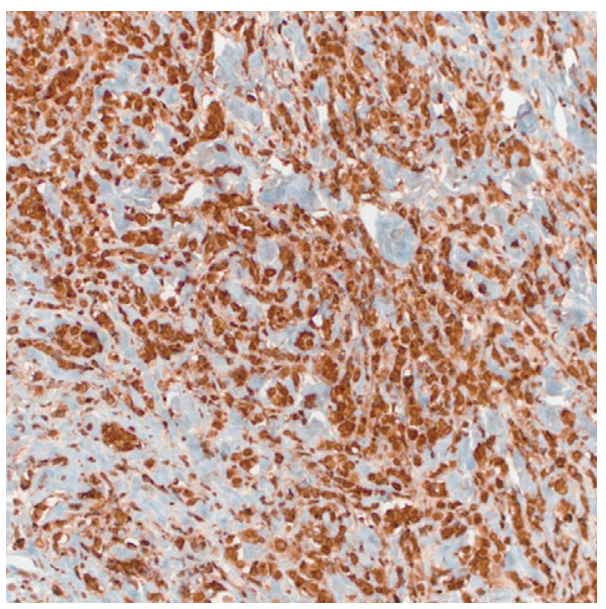

Figure 4. Immunohistochemical Stain, Positive for Mammaglobin.

guided biopsy of the breast mass with immunohistochemical studies confirmed the presence of invasive lobular carcinoma of the breast. Further imaging studies including a bone scintigraphy excluded other metastasis. The patient was treated by hormonal therapy and has been under close surveillance.

\section{Discussion}

A variety of tumors and pseudotumors can involve the orbit and approximately one-third of all orbital tumors are malignant. The likelihood of malignancy tumors increases with age, since older patients are more prone to metastasis. A large retrospective study reviewing over 1,200 patients referred for orbital mass reported lymphoid neoplasia (11\%), idiopathic orbital inflammation (11\%), and metastatic breast cancer $(4 \%)$ as some of the common diagnoses. Regarding metastases, breast cancer is the most common primary source of solid organ malignancy (1).

In this case, the initial clinical breast examination failed to detect the cancer. Mammography was also indefinite to 
detect malignancy in this patient. The detection of invasive lobular carcinoma (ILC) by screening mammography is known to be difficult and false negative rates have been reported to range from $8-19 \%$ (2), although ILC is the second most common type of invasive breast cancer after invasive ductal carcinoma (IDC) $(3,4)$. ILC arises from the lobules of the breast and it is known to be more often multifocal, multicentric and poorly delimited. A discrete mass is less common and a higher incidence of subtle mammographic changes, such as asymmetric density or architectural distortion has been reported. ILC has a less characteristic mammographic pattern than IDC and more frequently involves unusual sites such as the orbit, the gastrointestinal tract and the meninges (5). The most likely explanation for the false negative detection is that ILC spreads through the breast parenchyma by means of diffuse infiltration of single rows of malignant cells in a linear fashion around non-neoplastic ducts, which causes little disruption of the underlying anatomic structures and generates little surrounding connective tissue reaction.

In evaluating orbital masses, magnetic resonance imaging (MRI) has become an essential diagnostic tool. Advantages of MRI over computerized tomography (CT) include a higher soft tissue contrast and the lack of ionizing exposure. MRI is the imaging method of choice in diagnoses of orbital tumor. However, the availability of MRI should not negate the necessity of obtaining a careful history and performing a comprehensive physical and ocular examination. This patient had ophthalmoparesis due to neoplastic infiltration into the extrinsic muscles and the surrounding tissues of the right ocular bulb. Usually, metastasis to the uvea is more common than metastasis to the orbit or periorbital structures $(6,7)$. Orbital imaging studies and subsequent biopsy of the orbit together with the ascites fluid analysis lead to the diagnosis of ILC. The challenging aspect of this case is the bland and deceptive nature of the ILC tumor cells. The histopathologic characteristics of ILC are bland, dis-cohesive cells which are arranged individually within the fibrous connective tissue or arranged in single file rows invading through the stroma with very few mitoses. Also, ILC tumor cytology is usually bland with very few if any mitotic figures. ILC metastasis to fluids can be difficult to detect because the cells typically are small and round with occasional cells having plasmacytoid or histiocytic changes. Therefore, the ILC cells can resemble mesothelial cells, histiocytes, or lymphocytes in fluid cytology. Heightened awareness for detection of these cells is required so that immunohistochemical studies may be performed to make a definitive diagnosis.

The dispersed histologic pattern of ILC is likely to be related to its tumor biology, possibly involving the loss of the adhesion molecule E-cadherin in cancer cells; however, the factors governing the metastatic potential and tropism for a particular organ are complex and remain unclear. ILC is typically positive for estrogen receptor and progesterone receptor and negative for E-cadherin by immunohistochemistry. To define lobular differentiation of breast cancer, mor- phologic evaluation and supportive immunohistochemical findings are essential (8).

Treatment for metastatic breast cancer is usually palliative in intent. The goals of treatment include improving the quality and prolongation of life. Median survival has been reported to be 18 to 24 months (9). Treatment of metastatic breast cancer will usually involve hormone therapy and/or chemotherapy with or without monoclonal antibody therapy. Radiation therapy may be indicated for patients with limited symptomatic metastases. Hormone therapy generally should be considered as initial treatment for a postmenopausal metastatic breast cancer patient if the patient's tumor is estrogen receptor-positive or if the receptor status is unknown. While tamoxifen has been used in this setting for many years, several randomized trials suggest equivalent or superior response rates and progression-free survival with aromatase inhibitors. In a meta-analysis that included randomized trials in patients who were receiving an aromatase inhibitor as either their first or second hormonal therapy for metastatic disease, those who were randomly assigned to a third-generation drug (eg. Anastrozole) demonstrated longer survival (hazard ratio for death $=0.87 ; 95 \%$ confidence interval, 0.82-0.93) than those who received tamoxifen (10). Patients whose tumors have progressed on hormone therapy are candidates for chemotherapy. Patients with hormone receptor-negative tumors and those with visceral metastases are also candidates. At this time, no data support the superiority of any particular regimen. Whether single-agent chemotherapy or combination chemotherapy is preferable for first-line treatment is also unclear. Approximately $25 \%$ of patients with breast cancer have tumors that overexpress HER2/neu. Trastuzumab is a humanized monoclonal antibody that binds to the HER2/neu receptor. In a prospective trial, patients treated with chemotherapy plus trastuzumab had an overall survival advantage as compared with those receiving chemotherapy alone (25.1 months vs. 20.3 months, $\mathrm{p}=0.05)$ (11). The prognosis of patients with breast cancer with peritoneal metastases is poor (less than 2 months) (12).

In summary, we report a rare case in which orbital metastasis was the first sign of invasive lobular breast cancer. In the case of a metastatic orbital tumor, breast cancer should be considered as a possible source, especially in elderly women. Integrating the clinical information with histopathological findings is warranted.

\section{The authors state that they have no Conflict of Interest (COI).}

\section{References}

1. Shields JA, Shields CL, Scartozzi R. Survey of 1264 patients with orbital tumors and simulating lesions. Ophthalmology 111: 9971008, 2004.

2. Evans WP, Warren Burhenne LJ, Laurie L, Shaughnessy KF, Castellino RA. Invasive lobular carcinoma of the breast: mammographic characteristics and computer-aided detection. Radiology 225: 182-189, 2002.

3. Cristofanilli M, Gonzalez-Angulo A, Sneige $\mathrm{N}$, et al. Invasive 
lobular carcinoma classic type: response to primary chemotherapy and survival outcomes. J Clin Oncol 23: 41-48, 2005.

4. Pestalozzi BC, Zahrieh D, Mallon E, et al; International Breast Cancer Study Group. Distinct clinical and prognostic features of infiltrating lobular carcinoma of the breast: combined results of 15 International Breast Cancer Study Group clinical trials. J Clin Oncol 26: 3006-3014, 2008.

5. Lamovec J, Bracko M. Metastatic pattern of infiltrating lobular carcinoma of the breast: an autopsy study. J Surg Oncol 48: 2833, 1991.

6. Shields CL, Shields JA, Gross NE, Schwartz GP, Lally SE. Survey of 520 eyes with uveal metastases. Ophthalmology 104: 12651276, 1997.

7. Wickremasinghe S, Dansingani KK, Tranos P, Liyanage S, Jones A, Davey C. Ocular presentations of breast cancer. Acta Ophthalmol Scand 85: 133-142, 2007.

8. Chen YY, Rabban JT. Patterns of lobular carcinoma in situ and their diagnostic mimics in core needle biopsies. Pathology Case
Reviews 14: 141, 2009.

9. Kiely BE, Soon YY, Tattersall MH, Stockler MR. How long have I got? Estimating typical, best-case, and worst-case scenarios for patients starting first-line chemotherapy for metastatic breast cancer: a systematic review of recent randomized trials. J Clin Oncol 29: 456-463, 2011

10. Mauri D, Pavlidis N, Polyzos NP, et al. Survival with aromatase inhibitors and inactivators versus standard hormonal therapy in advanced breast cancer: meta-analysis. J Natl Cancer Inst 98: 12851291, 2006.

11. Slamon DJ, Leyland-Jones B, Shak S, et al. Use of chemotherapy plus a monoclonal antibody against HER2 for metastatic breast cancer that overexpresses HER2. N Engl J Med 344: 783-792, 2001.

12. Tuthill M, Pell R, Guiliani R, et al. Peritoneal disease in breast cancer: A specific entity with an extremely poor prognosis. Eur J Cancer 45: 2146-2149, 2009.

(C) 2012 The Japanese Society of Internal Medicine http://www.naika.or.jp/imindex.html 